\title{
A detailed quantum mechanical and quasiclassical trajectory study on the dynamics of the $\mathrm{H}^{+}+\mathrm{H}_{2} \rightarrow \mathrm{H}_{2}+\mathrm{H}^{+}$exchange reaction
}

\author{
Tomás González-Lezanaa and Octavio Roncero \\ Instituto de Matemáticas y Física Fundamental (CSIC), Serrano 123, 28006 Madrid, Spain \\ Pascal Honvault \\ LPM, UMR CNRS 6624, University of Franche-Comté, Campus de la Bouloie, 25030 Besançon Cedex, \\ France \\ Jean-Michel Launay \\ PALMS, UMR CNRS 6627, University of Rennes 1, Campus de Beaulieu, 35042 Rennes Cedex, France \\ Niyazi Bulut, ${ }^{\text {b) }}$ F. Javier Aoiz, and Luis Bañares \\ Departamento de Química Física I, Facultad de Química, Universidad Complutense, 28040 Madrid, Spain
}

(Received 2 June 2006; accepted 18 July 2006; published online 6 September 2006)

\begin{abstract}
The $\mathrm{H}^{+}+\mathrm{H}_{2}$ exchange reaction has been studied theoretically by means of a different variety of methods as an exact time independent quantum mechanical, approximate quantum wave packet, statistical quantum, and quasiclassical trajectory approaches. Total and state-to-state reaction probabilities in terms of the collision energy for different values of the total angular momentum obtained with these methods are compared. The dynamics of the reaction is extensively studied at the collision energy of $E_{\text {coll }}=0.44 \mathrm{eV}$. Integral and differential cross sections and opacity functions at this collision energy have been calculated. In particular, the fairly good description of the exact quantum results provided by the statistical quantum method suggests that the dynamics of the process is governed by an insertion mechanism with the formation of a long-lived collision complex. (C) 2006 American Institute of Physics. [DOI: 10.1063/1.2336224]
\end{abstract}

\section{INTRODUCTION}

The constantly renewed interest on the $\mathrm{H}_{3}^{+}$system has been recently manifested in a series of studies on a broad range of different aspects, which extends from dissociative recombination $^{1,2}$ to its experimental detection. ${ }^{3-5}$ The existence of very accurate $a b$ initio potential energy surfaces (PESs) corresponding to the ground and excited electronic states $^{6-11}$ has enabled both the theoretical calculation of its bound states ${ }^{12}$ and the study of the dynamics of atom-diatom collision processes involving the $\mathrm{H}_{3}^{+}$molecule. ${ }^{13-19}$ In particular, very recently, the dynamics of the $\mathrm{H}^{*}+\mathrm{D}_{2} \rightarrow \mathrm{HD}$ $+\mathrm{D}^{*}$ exchange reaction, where $\mathrm{H}^{*}$ is a Rydberg excited $\mathrm{H}$ atom, has been studied experimentally ${ }^{20-22}$ by means of the Rydberg H-atom translational spectroscopy technique. ${ }^{23,24}$ In these experiments, the hydrogen atom is highly excited to a Rydberg state, $\mathrm{H}(n \sim 35)$, before the reaction with deuterium molecules takes place. The long lifetime of the excited neutral D atoms allows to achieve extremely high resolution in the measured time-of-flight spectra. ${ }^{24}$ The comparison of the recorded rotational $^{22}$ and angular distributions ${ }^{20,21}$ with quasiclassical trajectory (QCT) results confirmed that the Rydberg electron effectively behaves as a "spectator" in the whole process. This enables us to describe the Rydbergatom+diatom reaction dynamics in terms of the ion + diatom exchange process.

\footnotetext{
a) Author to whom correspondence should be addressed: electronic mail: tglezana@imaff.cfmac.csic.es

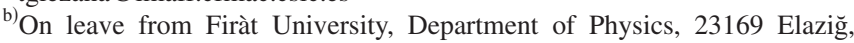
Turkey.
}

Despite the previous numerous occasions in which competing reactive and nonreactive processes with possible charge transfer between reactants have been theoretically studied, ${ }^{13-15,17-19,25-27}$ quantum mechanical (QM) results for nonzero total angular momentum $J$ for these reactions have not been reported until very recently. ${ }^{28,29}$ In these works, exact three-dimensional nonadiabatic QM calculations were carried out up to energies far above the electronic potential curve crossing and the three possible dynamical pathways [(i) the reactive charge transfer channel, which implies both reaction between the reactants and transfer of the charge; (ii) the nonreactive charge transfer channel, in which the charge migrates from the atom to the diatom; and (iii) the reactive noncharge transfer channel, which solely involves the atomdiatom reaction] were extensively investigated for both the $\mathrm{D}^{+}+\mathrm{H}_{2}$ (Ref. 28) and $\mathrm{H}^{+}+\mathrm{D}_{2}$ (Ref. 29) systems. QM approaches were also employed for the study of the reactive noncharge transfer pathway of these two collisions in a recent paper, ${ }^{30}$ where results from wave packet and statistical calculations were compared. It was found that the initial average description yielded by a statistical quantum method ${ }^{31,32}$ (SQM) for the wave packet $J>0$ reaction probabilities progressively deviates as the total angular momentum increases. In particular, although the reaction thresholds seemed to be correctly described by the SQM approach, intensities were clearly overestimated in comparison with the wave packet results. Nevertheless, the discrepancies inspired the suggestion of possible improvements for the usual centrifugal sudden (CS) approximation, ${ }^{33-35}$ which had been found to produce inaccurate results for the $\mathrm{D}^{+}+\mathrm{H}_{2}$ reaction. ${ }^{28,36}$ Thus, in 
addition to a cosine transformed quantum wave packet (QWP) method, a modified CS approach, where the centrifugal barrier was minimized, and an adiabatic approximation for the helicities were tested. In particular, the latter method, the adiabatic centrifugal sudden (ACS) approximation, was found to produce results in good accord with the SQM results for most of the total angular momentum values considered. $^{30}$

The application of the SQM seems, in principle, to be justified for insertion reactions, which are characterized by the formation of an intermediate complex between reactants and products. The existence of deep potential wells in these processes seriously complicates the use of exact quantum mechanical (EQM) methods. In this regard, previous comparisons with both experimental and EQM results on $X+\mathrm{H}_{2}$ reactions [where $X$ is a nonmetallic electronically excited atom as $\mathrm{C}\left({ }^{1} D\right), \mathrm{N}\left({ }^{2} D\right), \mathrm{O}\left({ }^{1} D\right)$ or $\left.\mathrm{S}\left({ }^{1} D\right)\right]$ have shown the adequacy of the method to tackle the dynamical study of such reactive collisions. ${ }^{31,32,37}$ Thus, it has been used as a reliable QM approach for systems where the substitution of one or two $\mathrm{H}$ atoms by a heavier isotope such as deuterium makes the EQM calculation computationally too expensive. $^{38,39}$ One of the goals of the present study is to extend the application of such model to the $\mathrm{H}^{+}+\mathrm{H}_{2}$ exchange reaction and to establish the possible discrepancies with the EQM results in terms of the previous findings on the $\mathrm{D}^{+}+\mathrm{H}_{2}$ and $\mathrm{H}^{+}+\mathrm{D}_{2}$ cases. ${ }^{30}$

In this work, the dynamics of the $\mathrm{H}^{+}+\mathrm{H}_{2}$ exchange reaction has been extensively investigated by means of time independent (TI) EQM, QWP, SQM, and QCT approaches. Special emphasis has been put in the specific collision energy $E_{\text {coll }}=0.44 \mathrm{eV}$, at which experimental research is currently in progress. ${ }^{40}$ Total and $v^{\prime}, j^{\prime}$ state-resolved integral and differential cross sections have been obtained at this collision energy. Comparisons between QM and experimental ${ }^{21}$ differential cross sections (DCSs) were reported for the $\mathrm{H}^{+}+\mathrm{D}_{2}$ reaction in Ref. 30. The theoretical total and stateto-state cross sections were obtained by means of the SQM approach at $0.53 \mathrm{eV}$ collision energy. The agreement found with experiment was similar to the previously achieved in Ref. 21, where the theoretical DCSs were obtained by means of QCT calculations, at least for the limited angular range covered by the experimental results. In the present work, EQM DCSs for the $\mathrm{H}^{+}+\mathrm{H}_{2}$ process are reported for the first time in comparison with QCT and SQM results obtained on the $a b$ initio PES from Aguado et al. ${ }^{7}$ The paper is structured as follows: In Sec. II, the most relevant aspects of the theoretical approaches are described. In Sec. III, results are shown and in Sec. IV a final summary and conclusions are presented.

\section{THEORY}

In this section we review the theoretical methods employed to study the dynamics of the $\mathrm{H}^{+}+\mathrm{H}_{2}(v=0, j=0)$ exchange reaction. The PES used in the present calculations was, as in Ref. 30, the recently proposed adiabatic PES by Aguado et al. $^{7}$ The minimum of this PES $(\sim 4.3 \mathrm{eV})$ corresponds to an equilateral configuration for an equilibrium bond length of $1.65 a_{0}$. The barrier for linear geometries, on the other hand, is located about $1.86 \mathrm{eV}$. The large depth of the PES and the lack of barriers in the entrance channel may suggest, in principle, that the reaction proceeds via an insertion mechanism characterized by the existence of long-lived resonances. Nonadiabatic effects and contributions from the triplet states are expected for energies about $1.6 \mathrm{eV}$ above the $\mathrm{H}_{2}$ well minimum, due to the crossing between the energy curves of states correlating to $\mathrm{H}^{+}+\mathrm{H}_{2}$ and $\mathrm{H}_{2}^{+}+\mathrm{H}$ at the asymptote. Nevertheless, for the smaller energy range considered in the present work, this would not constitute an issue.

\section{A. Time independent exact quantum method}

Details of the TI EQM method can be found in Ref. 41 and only a brief description is given here. This method has proven to be successful in describing the quantum dynamics of atom-diatom insertion reactions, such as $\mathrm{N}\left({ }^{2} D\right)+\mathrm{H}_{2},{ }^{42}$ $\mathrm{O}\left({ }^{1} D\right)+\mathrm{H}_{2},{ }^{43} \mathrm{C}\left({ }^{1} D\right)+\mathrm{H}_{2},{ }^{44}$ and ultracold alkali-atom-alkalidimer collisions. ${ }^{45-47}$ Nuclear motions in the $\mathrm{H}_{3}^{+}$system are represented by a set of coordinates which are a modified version of the Smith-Whitten democratic coordinates. They consist of three Euler angles, representing the orientation of the triatomic system in space, and three internal coordinates: the hyper-radius $\rho$, which characterizes the size of the system, and two hyperangles, which characterize its shape. We first compute a set of surface state eigenfunctions of a fixed$\rho$ reference Hamiltonian $H_{0}=T+V$, which incorporates the total energy with the potential energy $V$ and the kinetic energy $T$ arising from deformation and rotation around the axis of least inertia. These adiabatic states are expanded onto a basis of pseudohyperspherical harmonics. At small $\rho$, the adiabatic states span a large fraction of configuration space and allow for atom exchange. At large $\rho$, they concentrate into the arrangement valleys. The size of the expansion basis varies between 1500 and 4000 depending on $\rho$ and $\Omega$ (where $\Omega$ is the projection of the total angular momentum $J$ on the axis of least inertia). The range of variation of the hyperradius is divided into 113 sectors between 0.5 and $15.5 a_{0}$. The surface states dissociate into the $\mathrm{H}_{2}(22,20,19,17,15$, $13,10,7)$ rovibrational sets (this notation indicates the largest rotational level $j$ for each vibrational manifold $v$ $=0,1, \ldots$, . We then perform a close-coupling expansion of the full $\mathrm{H}_{3}^{+}$wave function onto the surface states. The hyperradial components satisfy a set of second-order coupled differential equations. The logarithmic derivative matrix is propagated inside each sector using the JohnsonManolopoulos algorithm. ${ }^{48,49}$ Basis transformations are performed at the boundary between sectors, and at large hyperradius, the numerically integrated wave function is matched onto a set of regular and irregular asymptotic functions expressed in the space fixed frame. The $K$ and $S$ matrices are extracted, and reactive integral and differential cross sections are obtained from standard equations. Production runs were performed with all surface states with $|\Omega| \leqslant 22$ and all partial waves with $J \leqslant 43$ in the close-coupling expansion (198-1841 channels for $J=0-43)$. 


\section{B. Quantum wave packet method}

The QWP method was described previously in detail in Ref. 30. The wave packet is represented in reactant Jacobi coordinates in a body-fixed frame: numerical grids are used for the internal coordinates, $r, R$, and $\gamma$, and parity adapted combinations of Wigner rotation matrices depending on three Euler angles to define a total wave packet with a good total angular momentum $J$. The details of grids are identical to those previously used. ${ }^{30}$ A modified Chebyshev propagator is used to propagate a real wave packet. ${ }^{51-56}$ The initial real wave packet consists of the product of the rovibrational state of the reactant, $\mathrm{H}_{2}(v=0, j=0)$ in the present case, times the real part of an incoming Gaussian function. ${ }^{56}$ In this approach the evolution operator is expanded in Chebyshev polynomials, modified by including a damping function for absorption at the edge of radial grids. ${ }^{52}$ Thus the Chebyshev real components of the wave packet, $\Phi_{k}$, are iteratively obtained and can be considered as a cosine transformed wave packet component. Once all $\Phi_{k}$ are obtained, the complex wave packet can be built at any time or the stationary eigenfunctions at any energy. Thus the total reaction probability is obtained by evaluating the flux as these Chebyshev components are being obtained as ${ }^{56}$

$$
\begin{aligned}
P_{R}(E)= & \frac{2}{m \pi\left|a_{\alpha}(E)\right|^{2}} \operatorname{Im} \sum_{\Omega} \int d R \int \sin \gamma d \gamma \\
& \times\left[\left.C_{n}^{*}\left(r_{\infty}, R, \gamma, E\right) \frac{d C_{\Omega}(r, R, \gamma, E)}{d r}\right|_{r=r_{\infty}}\right],
\end{aligned}
$$

where $m$ is the reduced mass of the reactant diatomic fragment, $r_{\infty}$ corresponds to the value of $r$ where the flux is evaluated, and

$$
C_{\Omega}(r, R, E)=\sum_{k=0}^{\infty} c_{k}\left(\hat{H}_{s}, E\right) \Phi_{\Omega}(r, R, \gamma ; k)
$$

[please note that Eqs. (9) and (10) of Ref. 30 are wrong and should be replaced by these two equations]. $\Phi_{\Omega}(r, R, \gamma ; k)$ is the component for the helicity projection $\Omega$.

Several calculations including an increasing number of helicities were performed up to the case in which the total reaction probability did not increase significantly, and in Ref. 30 it was found that inclusion of $\Omega=0,1,2, \ldots, 10$ seemed to be enough to converge the reaction probabilities for the $\mathrm{H}^{+}+\mathrm{D}_{2}(v=0, j=0, J>30)$ collisions. However, the comparison of the opacity function with the exact TI quantum method (not shown here) at a fixed energy indicates that the convergence was not enough for $J>20$. To justify the lack of convergence of the QWP calculation in Ref. 30 it should be considered that at the energy of interest, relatively low rotational excitations of $\mathrm{H}_{2}$ fragments were open $\left(j_{\max }=7\right.$ at $\left.\mathrm{E}_{\text {coll }}=0.44 \mathrm{eV}\right)$. Therefore, in the entrance channel only low helicity projections $\left(\Omega \leqslant j_{\max }\right)$ are possible, and the inelastic probabilities would then be converged. $\mathrm{A}_{2}\left(j^{\prime}=7\right)$ product described using reactant Jacobi coordinates corresponds to a relatively broad expansion in spherical harmonics. Just as an example, an initial wave packet in the entrance channel, corresponding to $\mathrm{H}_{2}(v=0, j=7, \Omega=0$ and 7) times an incoming

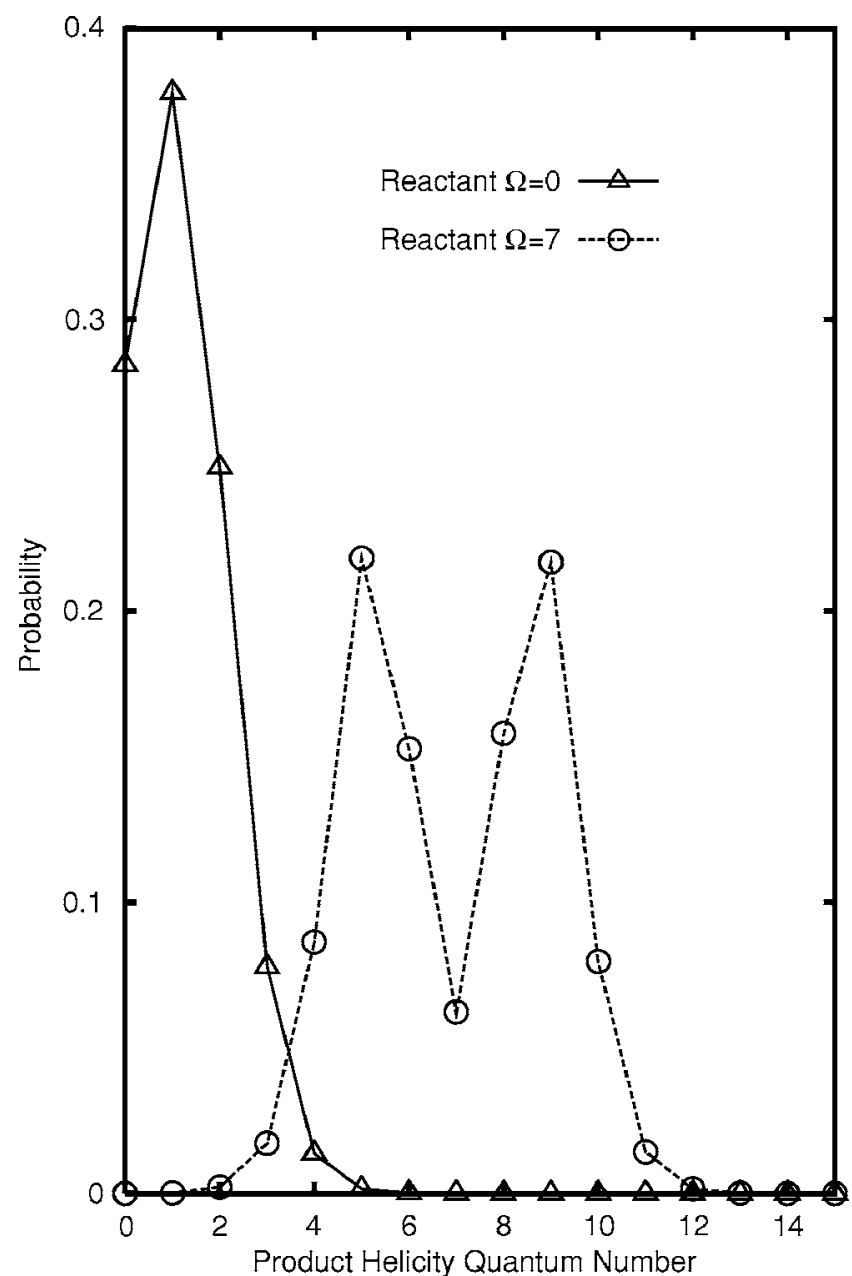

FIG. 1. Helicity distributions for an initial wave packet for $\mathrm{H}^{+}+\mathrm{H}_{2}(v=0, j$ $=7)$ at $J=30$ after transformation to product Jacobi body-fixed coordinates.

Gaussian centered at $R=12 \AA$, is transformed to product Jacobi body-fixed frame for total angular momentum $J=30$, and the resulting helicity projections are shown in Fig. 1. The product helicity distribution becomes broader, specially for $\Omega=7$. This fact may partially explain the disagreement between the exact TI and the QWP (with $\Omega=0,1,2, \ldots, 10$ ) calculations. The lack of convergence must be also due to the strength of the Coriolis coupling, which becomes very large in the vicinity of $R \approx 0$.

The inclusion of a higher number of helicity components makes virtually impossible to calculate the cross section for all total angular. In a wave packet calculation, this would involve a complete propagation for each partial wave. In Ref. 30 an alternative to the usual CS approach was proposed for insertionlike reactions: the ACS approximation. In this approach, only one helicity projection is used but the barrier is substituted by the lower eigenvalue of the $\hat{\ell}^{2}$ term at each grid point, with $\hat{\ell}$ being the operator associated with the endover-end angular momentum of $\mathrm{H}_{2}$ reactant with respect to $\mathrm{H}^{+}$and represented in a complete basis of $\Omega$ functions. The resulting barrier is lowered, leading to a much better description of the reaction threshold. In Ref. 30 the disagreement between the ACS and "exact" calculations was attributed to a failure of the ACS approach. In this work, it has been ob- 
served that the exact calculation was not converged and that the maximum number of $\Omega$ should be increased to get the converged TI results. Surprisingly, the ACS treatment gets much better results when compared with TI results, as will be discussed below, with a lower computational effort. Even when the results are not exact, specially for $J>35$ as will be shown below, it opens a new approach for development providing a nice physical insight of the reaction dynamics.

\section{Statistical quantum method}

The interested reader on the theoretical foundations of the SQM employed in the present work is referred to the first two original papers where the model was introduced ${ }^{31,32}$ and its recent applications to insertion processes. ${ }^{30,37-39}$ The crucial aspect to remark here is that the main assumption for the model to be valid is the need of the formation of an intermediate complex during the process of the atom-diatom reaction. The lifetime of such a collision complex is moreover considered to be long enough to justify the study of the whole process by treating its formation and subsequent fragmentation as independent events. Having fulfilled these requirements, it is then possible to approximate the state-tostate reaction probability between an initial $v j \Omega$ state in the arrangement $\alpha$ and a final $v^{\prime} j^{\prime} \Omega^{\prime}$ state in the arrangement $\alpha^{\prime}$, for a total angular momentum $J$ and a parity eigenvalue $I$, in the helicity representation as

$$
\left|S_{\alpha^{\prime} v^{\prime} j^{\prime} \Omega^{\prime}, \alpha v j \Omega}^{I J}(E)\right|^{2} \simeq \frac{p_{v^{\prime} j^{\prime} \Omega^{\prime}}^{I J \alpha^{\prime}}(E) p_{v j \Omega}^{I J \alpha}(E)}{\sum_{\alpha^{\prime \prime} v^{\prime \prime} j^{\prime \prime} \Omega^{\prime \prime}} p_{v^{\prime \prime} j^{\prime \prime} \Omega^{\prime \prime}}^{I J \alpha^{\prime \prime}}(E)},
$$

where $v$ and $j$ are the diatomic vibrational and rotational quantum numbers, respectively, and $\Omega$ is the modulus of the projection of the diatomic rotational angular momentum on the atom-diatom axis. In Eq. (3), $p_{v j \Omega}^{I J \alpha}$ and $p_{v^{\prime} j^{\prime} \Omega^{\prime}}^{I J \alpha^{\prime}}$ can be understood as the capture probability or probability of forming the collision complex from the reactant channel $v j \Omega$ and as the capture probability from the product channel $v^{\prime} j^{\prime} \Omega^{\prime}$, i.e., the probability for the collision complex to decay to that product channel, respectively. The capture probabilities can be written as

$$
p_{v j \Omega}^{I J \alpha}(E)=1-\sum_{v^{\prime} j^{\prime} \Omega^{\prime}}\left|S_{v^{\prime} j^{\prime} \Omega^{\prime}, v j \Omega}^{I J \alpha}(E)\right|^{2},
$$

where the sum runs over all the energetically open channels. Equation (4) expresses that the lack of unitarity of the scattering matrix $S^{J}(E)$ with respect to an usual inelastic scattering calculation is attributed to capture by the collision complex. ${ }^{31}$ The scattering matrix in the above expression is thus obtained by solving for each chemical arrangement within the CS approximation the corresponding closecoupled equations. ${ }^{31}$ The solution of those equations is performed by means of a TI log derivative propagation ${ }^{49}$ between the corresponding asymptotic region and a specific capture radius which defines the region where the complex is assumed to exist. In this case, the same region as the previous study of Ref. 30 on the $\mathrm{H}^{+}+\mathrm{D}_{2}$ and $\mathrm{D}^{+}+\mathrm{H}_{2}$ reactions between $3 a_{0}$ and $15 a_{0}$ for such propagation is chosen.
The statistical integral cross section (ICS) can be then evaluated from the EQM expression

$$
\begin{aligned}
\sigma_{\alpha^{\prime} v^{\prime} j^{\prime}, \alpha v j}(E)= & \frac{\pi}{g k_{\alpha v j}^{2}(2 j+1)} \sum_{I J \Omega \Omega^{\prime}}(2 J+1) \\
& \times\left|S_{\alpha^{\prime} v^{\prime} j^{\prime} \Omega^{\prime}, \alpha v j \Omega}^{I}(E)\right|^{2},
\end{aligned}
$$

with $k_{\alpha v j}^{2}=2 \mu\left(E-E_{v j}\right) / \hbar^{2}, g$ being the electronic degeneracy, and the reactive probability $\left|S_{\alpha^{\prime} v^{\prime} j^{\prime} \Omega^{\prime}, \alpha v j \Omega}^{I J}(E)\right|^{2}$ approximated as Eq. (3).

The expression for the statistical DCSs, once a random phase approximation is invoked, is the following: ${ }^{32}$

$$
\begin{aligned}
\sigma_{\sigma^{\prime} v^{\prime} j^{\prime}, \alpha v j}(\theta, E) \simeq & \frac{1}{8 k_{\alpha v j}^{2}} \frac{1}{2 j+1} \sum_{I J \Omega^{\prime} \Omega}(2 J+1)^{2} \\
& \times\left[d_{\Omega^{\prime} \Omega}^{J}(\pi-\theta)^{2}+d_{\Omega^{\prime} \Omega}^{J}(\theta)^{2}\right] \\
& \times\left|S_{\alpha^{\prime} v^{\prime} j^{\prime} \Omega^{\prime}, \alpha v j \Omega}^{I J}(E)\right|^{2},
\end{aligned}
$$

where $d_{\Omega^{\prime} \Omega}^{J}(\theta)$ is a reduced rotation matrix. ${ }^{50}$

\section{Quasiclassical trajectory method}

QCT calculations have been performed for the title reaction at the fixed collision energy of $0.44 \mathrm{eV}$ by running a batch of $2 \times 10^{5}$ trajectories following the procedures described in detail elsewhere. ${ }^{57}$ The integration step size in the trajectories was chosen to be $5 \times 10^{-17} \mathrm{~s}$. This guarantees a total energy conservation better than one part in $10^{4}$ and conservation of total angular momentum better than one part in $10^{6}$. The trajectories were started at a distance between the incoming atom and the center of mass of the diatomic of $10 \AA$. The rovibrational energies of the $\mathrm{H}_{2}$ reagent product molecules were calculated by semiclassical quantization of the action using the potential given by the asymptotic diatom limits of the PES. These rovibrational energies were fitted to Dunham expansions containing 20 terms [fourth power in $v+1 / 2$ and third power in $j(j+1)]$. The assignment of product quantum numbers $\left(v^{\prime}, j^{\prime}\right)$ was carried out by equating the classical rotational angular momentum of the product molecule to $\left[j^{\prime}\left(j^{\prime}+1\right)\right]^{1 / 2} \hbar$. With the (real) $j^{\prime}$ value so obtained, the vibrational quantum number $v^{\prime}$ was found by equating the internal energy of the outgoing molecule to the corresponding Dunham expansion. In the most common procedure, these real $v^{\prime}$ and $j^{\prime}$ values are rounded to the nearest integer, in what is named the histogramatic binning method. However, as it has been demonstrated in previous works, ${ }^{58,59}$ this rounding procedure may cause important discrepancies between the classical and quantum internal energy distributions, especially in the case of slightly endoergic or nearly thermoneutral channels of a reaction. Moreover, rovibrational channels which are closed quantum mechanically can be classically accessible due to this rounding method. This may cause important distortions in the classical rotational distributions of the highest vibrational levels accessed by the products when compared with the QM ones, and the effect will be larger for those product molecules characterized by large vibrational and rotational spacings. As in previous works, ${ }^{58,59}$ we have implemented a Gaussian-weighted bin- 
ning (GWB) procedure, originally proposed by Bonnet and Rayez. ${ }^{60}$ Briefly, a Gaussian function centered at the quantal action and with a given width has been used to weight the trajectories following the criterion that the closer the vibrational action of a given trajectory to the nearest integer, the larger the weighting coefficient for that trajectory. In particular, in the present work, we have used a full width at half maximum for the Gaussian functions of 0.1. As it has been shown recently, ${ }^{58,59}$ a much better agreement is obtained between the QM and QCT vibrational branching ratios and product rotational distributions when the GWB procedure is employed in the QCT calculations. A similar procedure can be applied to the rotational action; however, the resulting cross sections and reaction probabilities are indistinguishable with those obtained without GWB.

Using the GWB procedure, DCSs were calculated for every rovibrational state of the $\mathrm{H}_{2}$ product molecule as usual by the method of moment expansion in Legendre polynomials. The Smirnov-Kolmogorov test was used to decide when to truncate the series. Significance levels higher than $99 \%$ could be achieved by using $8-16$ moments, depending on the number of reactive trajectories available, ensuring good convergence, such that the inclusion of more terms did not produce any significant change.

The collision energy evolution of the reaction probability at different values of the total angular momentum $J=0,10$, $20,30,35$, and $40, P^{J}\left(E_{\text {coll }}\right)$, for the title reaction has been calculated by running batches of $10^{5}$ trajectories for each value of $J$ in the collision energy range of $0.005-1.6 \mathrm{eV}$ as described in Ref. 57 using the expression

$$
b=\frac{\hbar}{\mu v_{r}}[J(J+1)]^{1 / 2},
$$

where $b$ is the impact parameter of the trajectories and $\mu$ and $v_{r}$ are the $\mathrm{H}_{3}$ reduced mass and relative velocity, respectively. The calculation of the vibrationally state-resolved reaction probabilities for $J=0$ has been performed by the method of moment expansion in Legendre polynomials and employing the GWB procedure commented on above to assign final vibrational states. The integration step size and the initial distance between the incoming atom and the center of mass of the diatomic were the same as in the batch at fixed collision energy mentioned above.

\section{RESULTS AND DISCUSSION}

In this section, reaction probabilities and cross sections for the $\mathrm{H}^{+}+\mathrm{H}_{2}(v=0, j=0)$ exchange reaction will be shown. The precise study of the dynamics of the process at $J=0$ as a function of the collision energy is also extended to the reaction probabilities for $J>0$ values. Furthermore the $\mathrm{H}^{+}$ $+\mathrm{H}_{2}(v=0, j=0)$ exchange reaction has been investigated at the particular collision energy of $0.44 \mathrm{eV}$, for which there are experimental studies on the Rydberg-atom $\mathrm{H}^{*}+\mathrm{H}_{2}$ reaction in progress. ${ }^{40}$ In the present work, theoretical opacity functions, ICS and DCS, at fixed collision energy have been calculated by means of the methods described in the previous section.
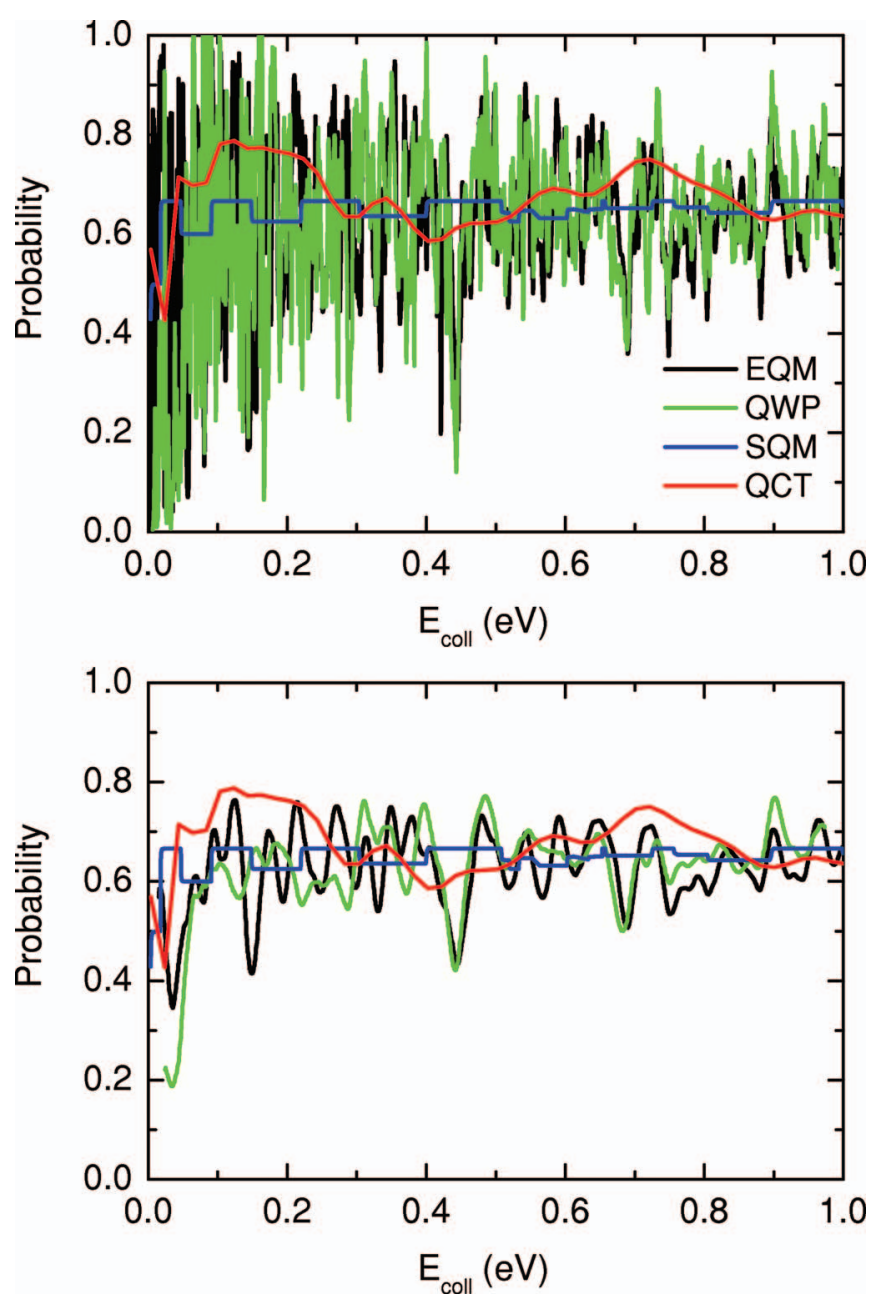

FIG. 2. (Color) Reaction probabilities for the $\mathrm{H}^{+}+\mathrm{H}_{2}(v=0, j=0)$ reaction and $J=0$ as a function of collision energy, $P\left(E_{\text {coll }}, J=0\right)$, obtained with the different theoretical methods employed in this work (top panel). EQM result is in black, QWP probability is in green, the SQM result is in blue, and the QCT result is in red. At the bottom panel, the same comparison is shown with the EQM and QWP probabilities smoothed by means of an energy convolution with a Gaussian function.

\section{A. Reaction probabilities}

The dynamics of the $\mathrm{H}^{+}+\mathrm{H}_{2}(v=0, j=0)$ exchange reaction has been initially probed by inspection of the reaction probability for total angular momentum $J=0$ as a function of collision energy. Figure 2 shows, at the top panel, the EQM, QWP, SQM, and QCT total reaction probabilities for $J=0$ for the collision energy range up to $1 \mathrm{eV}$. Both exact QM approaches yield reaction probabilities which are characterized by a large number of very narrow peaks. Apart from slight shifts in some of the resonance peaks, both methods provide reaction probabilities in a very good agreement. The fact that the reaction is mediated by a large number of very narrow resonances may be expected from the existence of a deep potential well. The QCT and SQM approaches produce, on the other hand, a fairly good average description of the quantum reaction probabilities. As already mentioned in previous studies, ${ }^{32}$ the SQM method, as the QCT method, cannot reproduce any resonance structures of the collisional process under study. In the SQM case, the reason is that the simulated statistical reaction probability involves an energy 
average $^{61}$ which precludes the model to provide any information of the possible existing resonances. The only appreciable structure of the SQM reaction probability has its origin in the gradual energetic opening of the different diatomic rovibrational states. ${ }^{30}$ In particular, the SQM reaction probability profile suffers a sudden increase every time a product channel state $v^{\prime}, j^{\prime}, \Omega^{\prime}$ becomes energetically accessible. Analogously, the opening of reactant channel states $v, j, \Omega$ produces a decrease on the statistical probability. Nevertheless, this "average" agreement yields information about the insertionlike nature of the reaction for $J=0$ as in the previously studied cases of the $\mathrm{H}^{+}+\mathrm{D}_{2}$ and $\mathrm{D}^{+}+\mathrm{H}_{2}$ reactions. ${ }^{30} \mathrm{As}$ expected, the QCT reaction probabilities do not show any sharp peak structure, although they show broad oscillations, which follow approximately the average EQM and QWP reaction probabilities.

In order to facilitate the comparison with both the EQM and QWP results, probabilities obtained with these two methods have been smoothed by means of an energy convolution with a Gaussian function. As shown at the bottom panel of Fig. 2, once the structure of the exact result formed by the many narrow resonances existing in this reaction is washed out, the average description of both QCT and SQM approaches manifests more clearly.

A similar degree of agreement is also found among the EQM, QWP, SQM, and QCT methods for the vibrationally state-resolved $J=0$ reaction probabilities as shown in Fig. 3, where the $P_{v^{\prime}}\left(E_{\text {coll }}\right)$ reaction probabilities for $v^{\prime}=0, v^{\prime}=1$, and $v^{\prime}=2$ are compared. Interestingly, the $v^{\prime}=1$ and $v^{\prime}=2$ reaction probabilities show a threshold. The decrease in reaction probability for $v^{\prime}=0$ at around $0.5 \mathrm{eV}$ collision energy is due to the opening of the $v^{\prime}=1$ channel. The EQM and QWP thresholds are fairly well reproduced by the SQM and QCT approaches. In the QCT result, this is the case when the GWB procedure is employed, whereas for the histogramatic binning, the predicted QCT thresholds are significantly smaller than those obtained from the EQM and QWP methods.

Figure 4 shows the collision energy dependence of the reaction probability for the $\mathrm{H}^{+}+\mathrm{H}_{2}(v=0, j=0)$ exchange reaction obtained for selected values of $J>0$. The theoretical results shown correspond to the ACS-QWP, SQM, and QCT approaches. As $J$ increases, the reaction probability shows an increasing threshold. For low values of $J$, the three methods yield similar results, but for large $J$ 's, especially for $J=40$, the ACS and QCT reaction probabilities clearly underestimate the reactivity in comparison with SQM.

\section{B. Integral and differential cross sections at $0.44 \mathrm{eV}$ collision energy}

A further analysis of the reaction dynamics of the $\mathrm{H}^{+}$ $+\mathrm{H}_{2}(v=0, j=0)$ exchange reaction has been made by making calculations at the specific collision energy $E_{\text {coll }}=0.44 \mathrm{eV}$.

Vibrationally resolved ICSs at this collision energy have been calculated using the EQM, SQM, and QCT methodologies. Figure 5 shows the rotational distributions corresponding to the $\mathrm{H}_{2}\left(v^{\prime}=0, j^{\prime}\right)+\mathrm{H}^{+}$products. Interestingly, the EQM cross sections display an oscillatory behavior up to $j^{\prime}=4$,
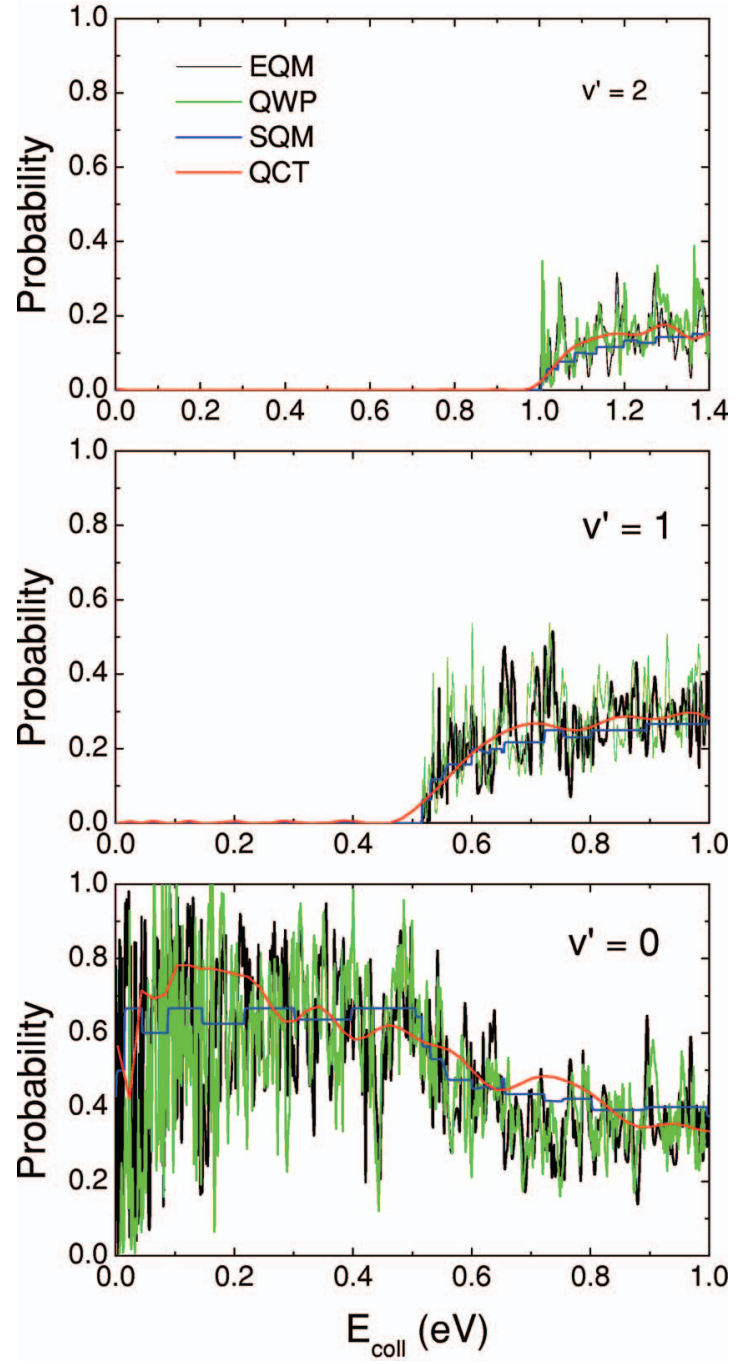

FIG. 3. (Color) Final vibrational-state-resolved reaction probabilities for the $\mathrm{H}^{+}+\mathrm{H}_{2}(v=0, j=0) \rightarrow \mathrm{H}^{+}+\mathrm{H}_{2}\left(v^{\prime}\right)$ reaction and $J=0$. Results for the $\mathrm{H}_{2}\left(v^{\prime}=0\right)$ case are shown at the bottom panel, $v^{\prime}=1$ at the middle panel, and $v^{\prime}=2$ at the top. Colors assigned to the EQM, QWP, SQM, and QCT methods as in Fig. 2.

with particularly low values for the cross sections for $j^{\prime}=1$ and $j^{\prime}=3$ in comparison with $j^{\prime}=0$ and $j^{\prime}=2$. The SQM prediction gives a fairly good description of the EQM results with the only exception of the ICS at those two $\mathrm{H}_{2}\left(v^{\prime}=0\right)$ rotational states $\left(j^{\prime}=1\right.$ and $\left.j^{\prime}=3\right)$. Those $j^{\prime}$ values are, on the contrary, the two rotational states for which the QCT cross sections get closer to the EQM results. The QCT calculations provide small values of the ICS for most of the rotational states which nevertheless describes well the minima of the EQM distribution $\left(j^{\prime}=1\right.$ and 3$)$. The disagreement for the rest of final $\mathrm{H}_{2}$ rotational states is closely related with the noticeable difficulties found for the QCT method to reproduce the reaction probabilities at the larger values of the total angular momentum $(J>20)$, as will be further discussed in Sec. III C.

The value of the total ICS at this collision energy also reflects the different degree of agreement found among the theoretical methods employed in this work. Whereas the SQM value, $23.70 \AA^{2}$, is in a good agreement with the EQM 

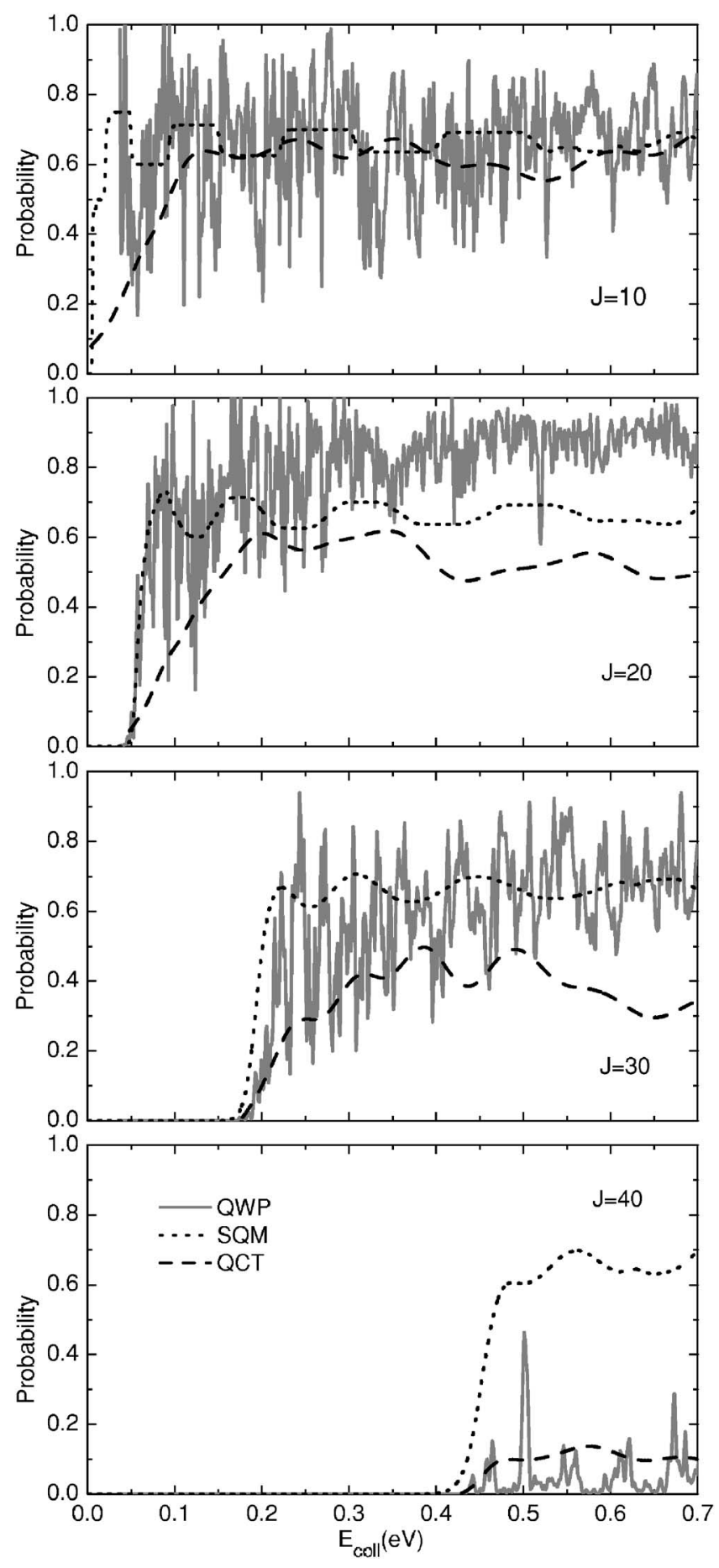

FIG. 4. Reaction probabilities $P\left(E_{\text {coll }}, J=10,20,30\right.$, and 40$)$ for the $\mathrm{H}^{+}$ $+\mathrm{H}_{2}(v=0, j=0)$ reaction obtained by means of the ACS-QWP (in gray solid line), SQM (black dotted line), and QCT (black dashed line) approaches.

value, $23.13 \AA^{2}$, the QCT and ACS-QWP predictions turn out to be significantly smaller, 15.23 and $16.5 \AA^{2}$, respectively.

Figure 6 shows the total DCS calculated for the title reaction at the collision energy of $0.44 \mathrm{eV}$ using the EQM, SQM, and QCT methodologies. The present EQM DCSs are, to our knowledge, the first exact converged DCS reported so far for the $\mathrm{H}^{+}+\mathrm{H}_{2}(v=0, j=0)$ exchange reaction. The most remarkable feature of the present EQM DCS is the extremely peaked character in both forward and backward scattering

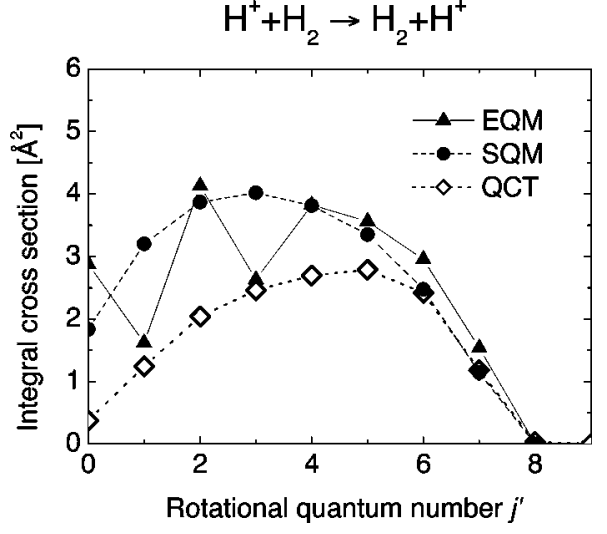

FIG. 5. Final rotational-state-resolved ICSs for the $\mathrm{H}^{+}+\mathrm{H}_{2}(v=0, j=0)$ $\rightarrow \mathrm{H}^{+}+\mathrm{H}_{2}\left(v^{\prime}, j^{\prime}\right)$ collisions at $E_{\text {coll }}=0.44 \mathrm{eV}$ obtained with the EQM method (black triangles and solid lines), SQM approach (black circles and dashed lines), and the QCT method (empty diamonds with dotted lines).

directions. In particular, the ratio of DCSs between extreme forward and sideways is more than 10. The SQM method manages to reproduce most of the main features of the EQM DCS. The backward peak and sideway scattering are satisfactorily reproduced by the SQM method; however, the statistical prediction clearly underestimates the forward peak. It must be emphasized that the SQM approach yields forwardbackward symmetric DCSs due to the random phase approximation employed. ${ }^{32,62}$ Thus, due to the slightly asymmetric character of the EQM DCS (a ratio of about 1.3 between the forward and backward peaks), this degree of agreement is by far more than one might expect between the statistical and exact QM approaches.

The QCT DCS, on the contrary, cannot reproduce the extreme forward and backward scattering although the sideways region of the DCS is successfully described. This apparent inability of the QCT method to describe extreme polarization effects between forward/backward and sideway scattering has also been found in some insertion reactions such as $\mathrm{C}\left({ }^{1} D\right)+\mathrm{H}_{2}$ (Ref. 58) and $\mathrm{S}\left({ }^{1} D\right)+\mathrm{H}_{2}{ }^{59}$ This sharp forward and backward peaked structure has been attributed to quantum effects such as tunneling through long range barriers. ${ }^{32}$ In this sense, it is interesting to note that attempts to include tunneling contributions through effective potential

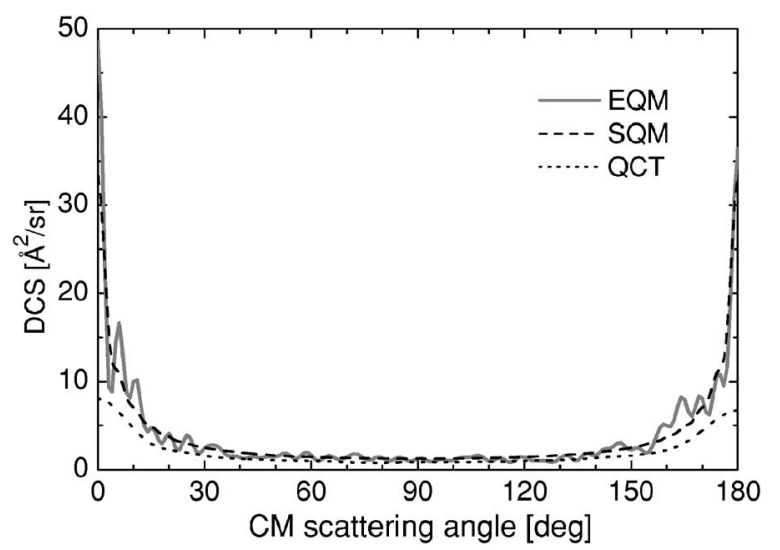

FIG. 6. Total DCSs for the $\mathrm{H}^{+}+\mathrm{H}_{2}(v=0, j=0)$ reaction at a collision energy of $E_{\text {coll }}=0.44 \mathrm{eV}$ calculated by means of the EQM (gray solid line), SQM (black dashed line), and QCT (black dotted line) methods. 


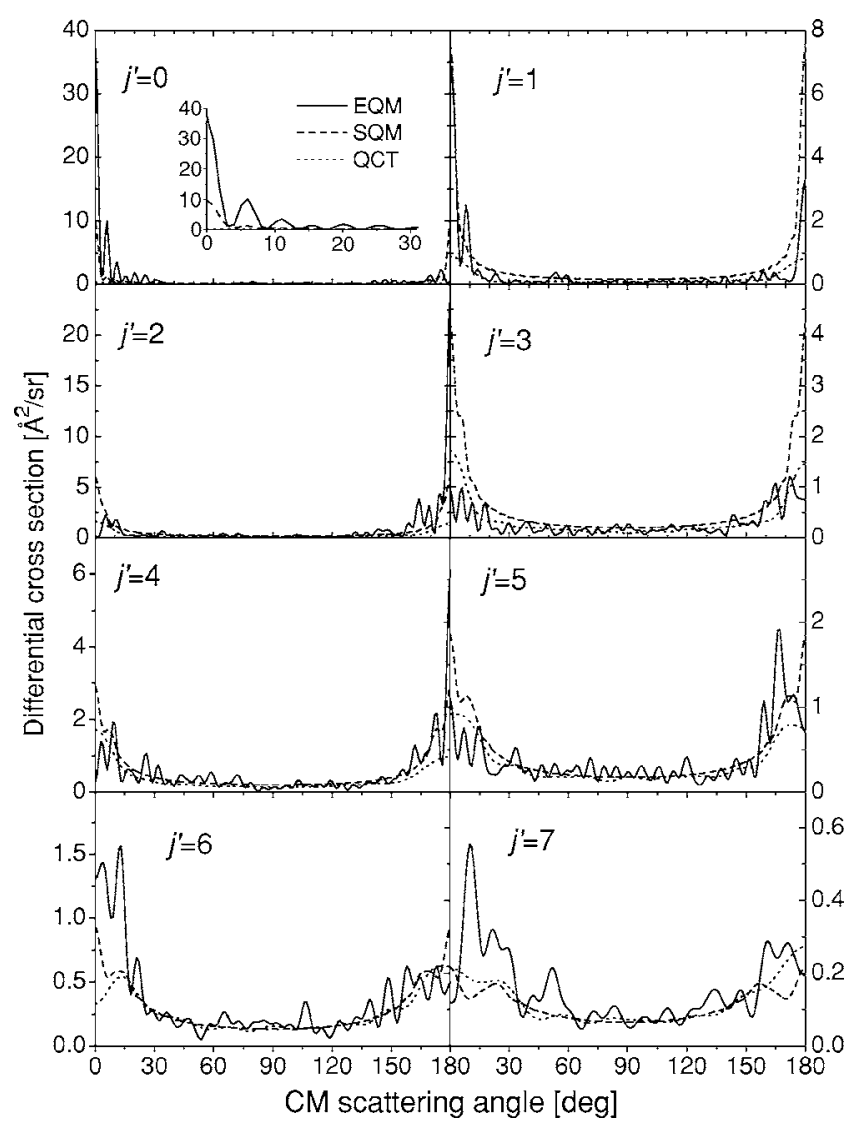

FIG. 7. Final rotational-state-resolved DCSs for the $\mathrm{H}^{+}+\mathrm{H}_{2}(v=0, j=0)$ $\rightarrow \mathrm{H}^{+}+\mathrm{H}_{2}\left(j^{\prime}\right)$ reaction (with $j^{\prime}=0-7$ ). Line types as in Fig. 6 .

barriers using the Wentzel-Kramers-Brillouin (WKB) semiclassical approximation have not solved the complete absence of this forward/backward dynamics in DCSs obtained in recent phase space theory calculations. ${ }^{63}$

Another interesting indicator to probe the dynamics of the title reaction is the fully state-resolved DCS. Figure 7 shows the EQM, SQM, and QCT rotationally state-resolved DCSs for the $\mathrm{H}^{+}+\mathrm{H}_{2}(v=0, j=0) \rightarrow \mathrm{H}_{2}\left(v^{\prime}=0, j^{\prime}\right)+\mathrm{H}^{+}$reaction calculated at $E_{\text {coll }}=0.44 \mathrm{eV}$. The structure of the rotationally resolved EQM DCSs shows a strong dependence on the specific value of $j^{\prime}$. The signature of a extremely peaked structure on both the forward and backward directions in the total DCS is present in the rotationally resolved DCSs for the lowest $j^{\prime}$ values. Thus, for $j^{\prime}=0-2$, the EQM DCSs show a clearly privileged scattering direction. The intriguing feature found in these state-to-state DCSs is that while for $j^{\prime}=0$ and $j^{\prime}=1$ forward scattering is preferred, for $j^{\prime}=2$ the situation is the opposite, with the DCS peaking at $\theta=180^{\circ}$. It is interesting to remark that this preference for the limiting cases $\theta=0^{\circ}$ or $\theta=180^{\circ}$ found in the state-to-state EQM DCSs occurs for those $j^{\prime}$ states for which the corresponding $j^{\prime}$ stateresolved opacity functions are clearly peaked at the higher values of the total angular momentum just before reaching the maximum value of $J$ (see Sec. III C). Although the DCS profiles are also asymmetric for some other $j^{\prime}$ states, the ratio between the prominent peak and the rest of the DCS is not so extreme as in the first three cases. In addition, the values of the DCSs at the extreme scattering angles are very large for $j^{\prime}=0$ and $j^{\prime}=2$ and significantly smaller for $j^{\prime}=1$, in accor-

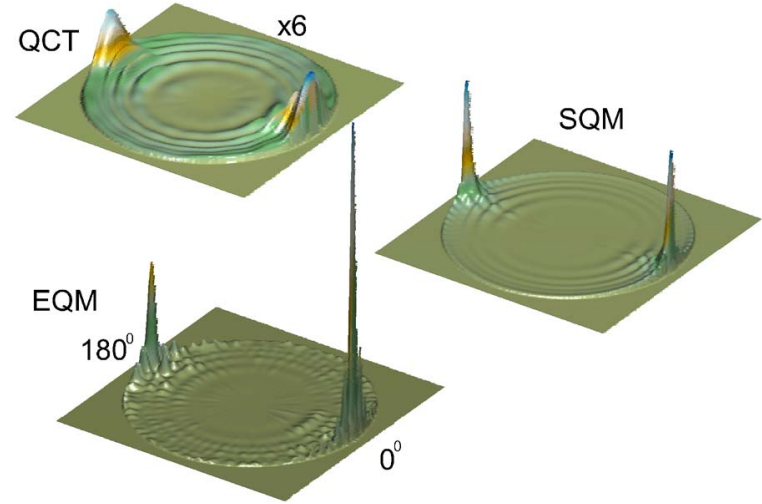

FIG. 8. Scattering angle-recoil velocity three-dimensional (3D) polar map for the $\mathrm{H}^{+}+\mathrm{H}_{2}(v=0, j=0)$ exchange reaction calculated at $E_{\text {coll }}=0.44 \mathrm{eV}$ using the EQM (left down), QCT (left top), and SQM (right middle) approaches. The $z$ axis of the QCT polar map has been multiplied by a factor of 6 .

dance with the evolution of the ICS with $j^{\prime}$ shown in Fig. 5. As $j^{\prime}$ increases, the EQM DCSs do not show strong peak structure at the extreme forward and backward directions, although an oscillatory behavior remains at all scattering angles.

The comparison of the SQM and EQM DCSs is very irregular. The highly nonsymmetric nature of the EQM DCSs makes the statistical prediction to reproduce only in part the exact result. Thus for $j^{\prime}=0-2$, where the EQM DCSs show the markedly peaked character mentioned above, the SQM approach is only capable to give a precise description at $\theta=0^{\circ}$. This implies that for $j^{\prime}=0$ and $j^{\prime}=2$ states, the statistical DCSs miss the dynamical feature present in the corresponding EQM DCSs. On the contrary, the comparison of the SQM and EQM DCSs for $j^{\prime}=1$ is fairly good at the forward direction, but the statistical peak at $\theta=180^{\circ}$ exceeds substantially the EQM result. The most unfavorable case for the SQM approach in comparison with the EQM method is $j^{\prime}=3$. This rotational state is the first one for which the EQM DCS misses any preference for either forward or backward scattering. The SQM prediction only manages to grasp some of the right angular behavior around the sideway direction. The agreement between the EQM and SQM DCSs improves in general for the rest of the $j^{\prime}$ states, between $j^{\prime}=4$ and $j^{\prime}=7$.

In the QCT case, the disagreement with the EQM DCSs is specially noticeable for $j^{\prime}=0$. For this rotational state, an amplified inset of the DCS for the $\theta=0^{\circ}-30^{\circ}$ scattering range is included in the left top panel of Fig. 7. It is for the forward direction of the DCS for the $j^{\prime}=0$ state where the largest discrepancies are found between the EQM, SQM, and QCT results. In particular, the QCT DCS is almost negligible in comparison with the EQM and SQM DCSs. Nevertheless, the agreement between QCT, EQM, and SQM DCSs gets much better as $j^{\prime}$ value increases.

A better way to visualize the agreements and disagreements between the DCSs obtained by the EQM, SQM, and QCT methods is by means of a scattering angle-recoil velocity polar map. Such polar maps are depicted for the title reaction in Fig. 8. As can be seen, the EQM polar map indicates that most of the scattering is concentrated in the ex- 

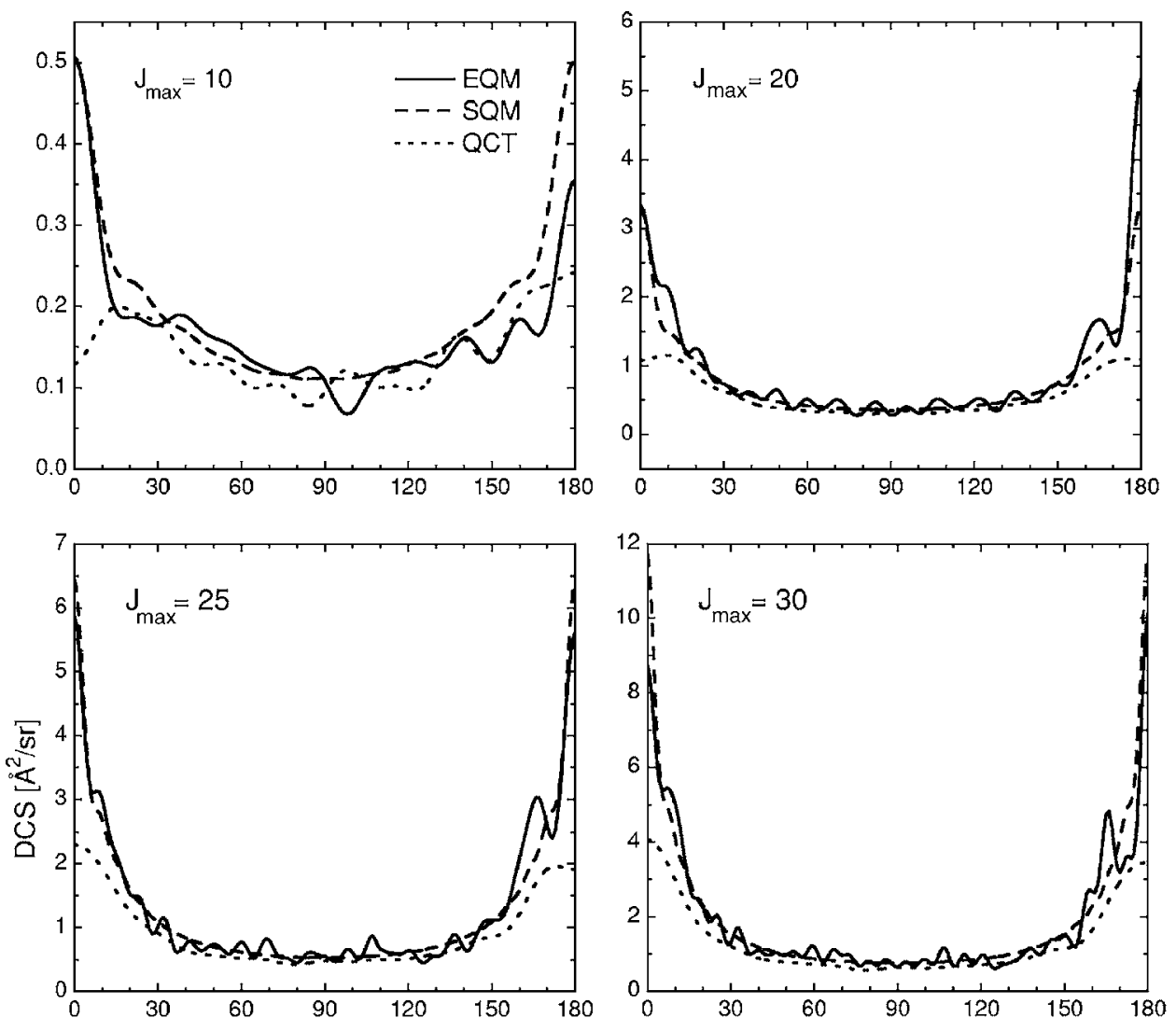

FIG. 9. Total DCSs for different maximum values $J_{\max }$ of the total angular momentum retained in the partial wave expansion for the $\mathrm{H}^{+}+\mathrm{H}_{2}(v$ $=0, j=0)$ collision at $E_{\text {coll }}=0.44 \mathrm{eV}$ obtained by means of the EQM, SQM, and QCT methods. Line types are as in Figs. 6 and 7.

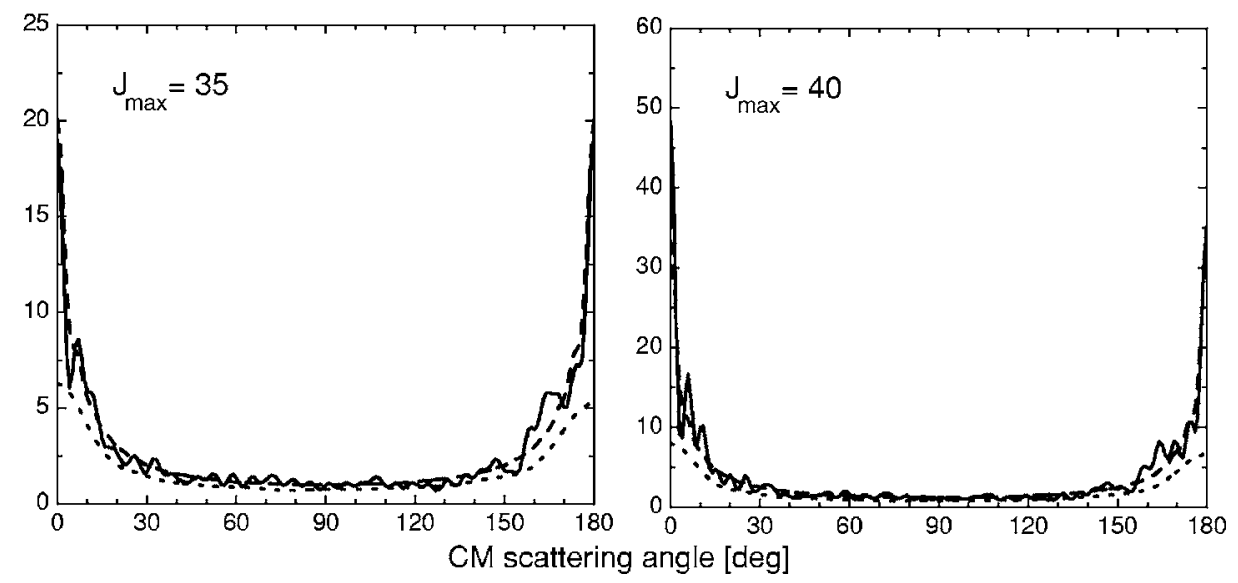

treme forward and backward directions in the form of very sharp peaks around $\theta=0^{\circ}$ and $\theta=180^{\circ}$ for the lowest product rotational states. The polar map is clearly dominated by the sharp peak at forward scattering and shows a lot of structure all around. The SQM polar map can reproduce most of the features of the EQM one, but the sharp peak at $\theta=0^{\circ}$ is significantly smaller. In addition, the scattering is smoother showing only some structure. The extreme case corresponds to the QCT polar map, which has been magnified in order to be compared with the EQM and SQM ones. In the QCT case, the scattering is also concentrated in the forward and backward regions but to a much lesser extent, since the forward and backward peaks are very small and sideway scattering competes favorably. No significant structures are observed in this case.

A clear indication of the important role played by the largest values of the total angular momentum $J$ on the dy- namics of the present reaction is found when we study the total DCS in terms of the maximum number of partial waves, $J_{\max }$, included for its calculation. In Fig. 9, total DCSs at $E_{\text {coll }}=0.44 \mathrm{eV}$ obtained by means of the EQM, SQM, and QCT methods are shown for $J_{\max }=10,20,25,30,35$, and 40. Interestingly, despite the forward-backward structure of the EQM, angular distribution is manifested even at the early stage of lower values of $J_{\text {max }}$; this particular dynamical feature is not dramatically exaggerated until the $J>35$ values of the total angular momentum start to contribute in the building up of the total DCS. In particular, the ratio between the forward-backward scattering direction and the sideway direction increases from a value close to 5 for $J_{\max }=10-25$ up to $\sim 10$ for $J_{\max }=35-40$. In addition the slight preference for the forward direction found for the total DCS at $E_{\text {coll }}$ $=0.44 \mathrm{eV}$ is not a constant for all partial waves. The statistical prediction, on the other hand, which probably repro- 


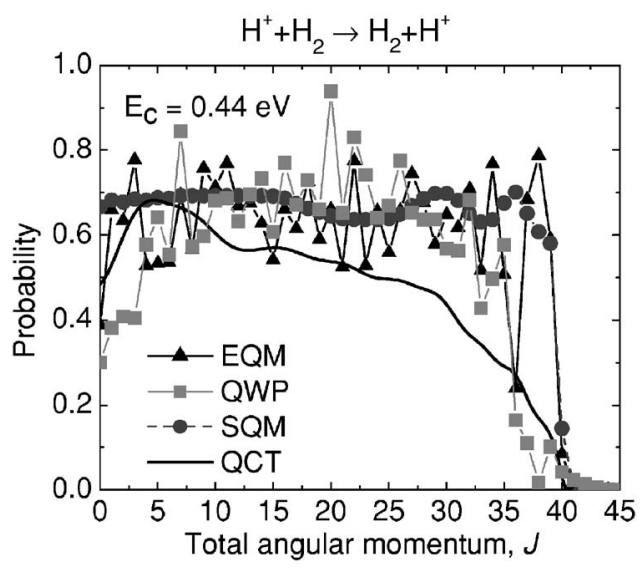

FIG. 10. Reaction probability as a function of the total angular momentum, $P\left(E_{\text {coll }}=0.44 \mathrm{eV}, J\right)$, at $E_{\text {coll }}=0.44 \mathrm{eV}$ for the $\mathrm{H}^{+}+\mathrm{H}_{2}(v=0, j=0)$ process. $\mathrm{EQM}$ results are shown in black line with triangles, ACS-QWP in light gray line with squares, SQM in dark gray line with circles, and QCT results are shown with black solid line.

duces more clearly the backward scattering peak when all partial waves are included, seems, however, to get closer to the forward maximum of the EQM result during the construction of the total DCS. Contribution of the largest values of $J$ makes the QCT DCS to improve somehow the ratio between forward-backward direction and sideway scattering but seems to be not enough to reproduce the QM results for any of the values of $J_{\max }$ considered here.

\section{Opacity functions}

Figure 10 shows the opacity function, i.e., the reaction probability as a function of total angular momentum $J$, calculated by means of the four theoretical methods described in Sec. II at $0.44 \mathrm{eV}$ collision energy. At this particular collision energy, the EQM probabilities show an oscillatory behavior around an average value close to 0.7 , which extends up to $J=40$, for which the total probability drastically decreases. Interestingly, the SQM method predicts both the right average trend of the EQM probability for the whole range of $J$ values and the final tail of the opacity function. This is a somehow unexpected agreement considering the conclusions previously obtained for the $\mathrm{H}^{+}+\mathrm{D}_{2}$ and $\mathrm{D}^{+}+\mathrm{H}_{2}$ isotopic variants. ${ }^{30}$

The ACS approach yields a rather good agreement with the EQM results up to $J=30$, as it is shown in Fig. 10. In the ACS calculation, the reaction probabilities were calculated for $J=0,5,10,20,25$, and 30 and for all $J$ values in the $(30,40)$ interval. For other $J$ values, the reaction probability was obtained by using a $J$-shifting-like interpolation. In Ref. 30 , it was concluded that the ACS approach overestimates the reaction probability with respect to the "nonconverged" QWP results. Since these last results yielded too low reactivity, we want to stress here the considerable improvement introduced by the ACS approximation at a much lower computational effort. The ACS approach fails, however, to describe reaction probabilities for $J>35$, as shown in Fig. 10 . The situation turns to be even more dramatic in the QCT case. Actually, the QCT opacity function only compares reasonably well with the EQM one for low values of $J$ and, in particular, for $J$ values $(21,23,36$, and finally 40$)$ which correspond to minima in the oscillations of the EQM reaction probability.

The main difference among the various methods occurs at large $J$ values. The SQM describes the overall EQM reaction probability very well but without showing the oscillations given its statistical character. The ACS approach shows oscillations and reproduces the reaction thresholds, but for $J>35$ this method is not able to reproduce the magnitude of the EQM reaction probability. The QCT method does not reproduce the oscillations either, and it underestimates the reaction probability with the only exception of low values of $J$.

In order to understand the origin of these differences, we concentrate now on the collision energy dependence of the reaction probabilities at different values of $J$ shown in Fig. 4. The three methods predict nearly the same reaction threshold, the differences among them corresponding essentially to the magnitude of the reaction probability. These differences are attributed to how each method treats the dynamics in the appearance of high rotational barriers. At low $J$ values, the dynamics is governed by Feshbach resonances due to the deep well. As $J$ increases, these resonances transform in orbiting resonances supported by increasing rotational barriers. The QCT method does not consider tunneling through the barrier, thus yielding a much lower reaction probability. The ACS treatment considers the tunneling but on an approximated barrier. The consideration of one single helicity projection with an effective adiabatic rotational barrier seems to be rather good until $J=35$. For higher angular momenta, however, it is not good enough probably due to two factors: first, when considering several helicities the density of resonances increases, and, second, when considering their mutual coupling their width also increases.

The reaction probabilities calculated within the statistical approximation as in Eq. (3) involve a nondiagonal product of capture probabilities corresponding to different helicities $\Omega$. Such terms justify the ACS approach and consider that once the complex is formed, the Coriolis coupling term is so efficient that the dynamics lose the "memory" of the initial $\Omega$. If this would not be the case and the $\Omega$ quantum number is maintained along the reaction dynamics, the sum in Eq. (3) should restrict to $\Omega=\Omega^{\prime}$. The results obtained doing this restriction (not shown here) yield a much lower reactivity. This enormous difference justify how important is to consider an adiabatic passage among the different $\Omega$ projections to follow the minimum energy path, as it is considered in the ACS approach. At the same time it provides a clue for the failure of considering a single helicity in the ACS method: In order to get higher reaction probabilities, some transitions among different helicities should be considered by including more adiabatic channels. Some work on this line is now in progress.

Figure 11 shows the $v^{\prime}, j^{\prime}$ state-resolved opacity functions calculated at $0.44 \mathrm{eV}$ collision energy by means of the EQM, SQM, and QCT methodologies. The EQM results show that the reaction reaches appreciable values up to $J=40$ for $j^{\prime} \leqslant 3$. For larger values of the final rotational state, a pronounced reduction of the maximum total angular mo- 


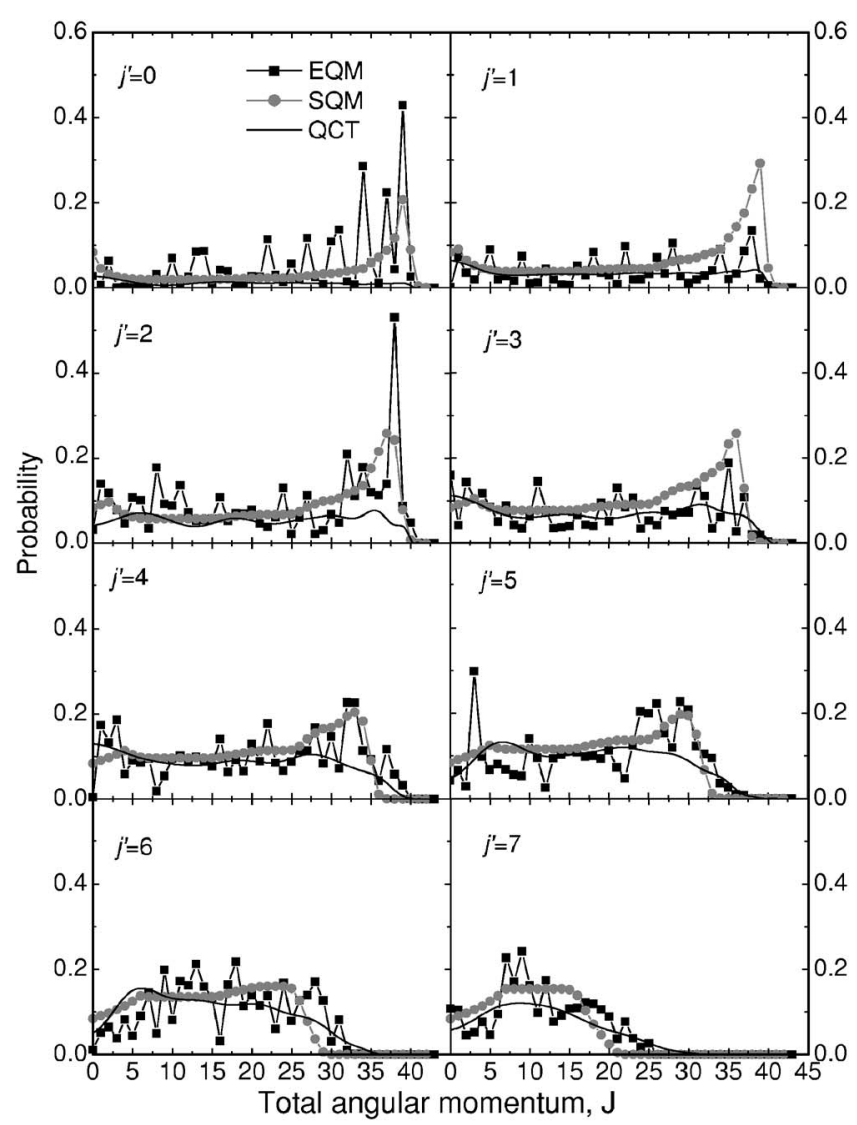

FIG. 11. Same as Fig. 10 but for the final rotational state $j^{\prime}$ resolved $\mathrm{H}^{+}$ $+\mathrm{H}_{2}(v=0, j=0) \rightarrow \mathrm{H}^{+}+\mathrm{H}_{2}\left(v^{\prime}=0, j^{\prime}\right)$ reaction. In this case EQM results are in black squares, SQM in gray circles, and QCT probabilities in solid line.

mentum accessible is observed. The general trend of the reaction probability seems to indicate an almost constant value on average for low and medium values of the total angular momentum $(J \leqslant 30)$ followed by a sudden increase which abruptly ends at the maximum value of $J$. This behavior is more clearly manifested in the cases of $j^{\prime}=0$ and $j^{\prime}=2$ for the EQM method and in almost all final rotational states $j^{\prime}$ in the SQM predictions.

The results shown in Fig. 11 indicate that in spite of following the overall dependence of the EQM reaction probability in terms of $J$, the state-to-state SQM opacity functions fail to provide a precise description of the final increase of the EQM probabilities before reaching the maximum value of $J$. Whereas the statistical method seems to overestimate the EQM result (see for instance right panels of first and second rows for $j^{\prime}=1$ and $j^{\prime}=3$, respectively), in some other cases $\left(j^{\prime}=0\right.$ and $\left.j^{\prime}=2\right)$ the SQM probabilities around that final peak of the opacity functions are smaller than the EQM result. In view of the previously mentioned good agreement found for the total opacity function (see Fig. 10), it is then interesting to notice how this apparent impossibility to reproduce strictly all fine details of the state-to-state dynamics of the reaction in a statistical context seems to be in some way mitigated when the reaction probability is obtained by summing over all final rotational states.

The QCT opacity functions are also included in Fig. 11. The comparison with the EQM probabilities is not extremely good for low values of $j^{\prime}$. In particular, for $j^{\prime}=0$ the QCT opacity function is almost negligible, in contrast with both EQM and SQM probabilities. For $j^{\prime}=3$ the situation improves considerably and the QCT prediction reproduces the EQM opacity function more satisfactorily than the statistical model. In addition, the tail of the exact opacity functions for the largest values of $j^{\prime}$ is better described by the QCT approach than by the SQM methodology, which seems to yield smaller values of the last accessible total angular momentum $J$.

The major differences among all the methods considered arise for $J>30$ and for $j^{\prime}<3$, as can be seen for the total or state-resolved opacity functions in Figs. 10 and 11. In those cases, the exact opacity function shows a maximum, which is probably the origin of the discrepancies found at small and large angle in the DCSs. Such maxima are attributed to resonances due to the barrier, either below (orbiting) or above (over barrier) the top of the barrier. These resonant structures are evident in the reaction probabilities obtained at high $J$, as the $J=40$ case shown in Fig. 4, using the ACS approximation but with a much lower probability. In order to get results in closer agreement with the EQM ones, those resonances should become much broader in energy, what could be obtained by including more helicity channels, increasing then the density of states, as commented above. Even when the ACS approach underestimates the width of such resonances appearing at high $J$, it can be used to get an idea of what is expected as a function of energy. In this sense, calculations by means of the ACS approximation of the opacity functions at slightly different collision energies (not shown here) reveal that the resonant structure near the limiting value of $J$ is strongly affected by those variations in energy. As a consequence, the previously discussed dynamical features found for the DCSs (as its possible forward/backward character) may change with energy. Experimental detection of such effects would depend on the actual width of those resonances with energy.

\section{SUMMARY AND CONCLUSIONS}

In this work, the exchange $\mathrm{H}^{+}+\mathrm{H}_{2}(v=0, j=0)$ reaction has been extensively studied by means of different theoretical quantum and quasiclassical methods. Reaction probabilities as a function of collision energy have been obtained for $J \geqslant 0$. Integral and differential cross sections at $E_{\text {coll }}$ $=0.44 \mathrm{eV}$ have been calculated for a possible future comparison with experiment.

The results of the present theoretical study on the title reaction suggest that the reaction is mainly dominated by an insertion mechanism in which the ion $\mathrm{H}^{+}$forms an intermediate complex in its collision with the $\mathrm{H}_{2}$ diatom. A first indication of such dynamics may be extracted from the large number of extremely narrow resonances in the reaction probabilities. Moreover, the overall good agreement between the EQM results and the SQM predictions reveals that this insertion pathway, favored by the existence of a deep potential well, has to play a key role in the process. Another feature in this direction is given by the structure of the total DCS of the reaction. The closely symmetric forward/backward scattering is a clear indication of the formation of such an intermediate 
complex. This characteristic shape has been related to the existence of long-lived collision complexes during the occurrence of reactive processes. ${ }^{64,65}$ Furthermore, the DCS found for the $\mathrm{H}^{+}+\mathrm{H}_{2}(v=0, j=0)$ reaction is extremely peaked in both the forward and backward directions, close to the typical $1 / \sin \theta$ behavior displayed in the classical limit of a model which assumes that the long lifetime of the intermediate complex results on the loss of all memory about the initial conditions of the reactant fragments. ${ }^{64}$ Noticeably, this dynamical feature seems to be compatible for the title reaction with extremely asymmetric $\left(v^{\prime}, j^{\prime}\right)$-state-resolved DCSs for some particular values of $j^{\prime}$. Animation of trajectories from the QCT calculations shows that all trajectories are of the insertion type. The distribution of collision times of the trajectories are also typical of a pure insertion reaction, similar to that found for instance for the $\mathrm{S}\left({ }^{1} D\right)+\mathrm{H}_{2}$ reaction. ${ }^{59}$

Previous studies on isotope arrangements of the title reaction ${ }^{14,66-68}$ have concluded that the dynamics of these processes evolves from a short-lived collision intermediate mechanism below about $3 \mathrm{eV}$ to a predominantly direct impulsive reaction pathway at larger energies $\left(E_{c}>4.5 \mathrm{eV}\right)$. Complex formation cross sections and lifetimes of those intermediate complexes were found to decrease rapidly as the collision energy increases. ${ }^{14,68}$ Although the same evidences have not been conclusively reported for the $\mathrm{H}^{+}+\mathrm{H}_{2}$ reaction, ${ }^{69,70}$ the long-lived complex dynamics at a low energy regime suggested by our present results seems to be consistent with this mentioned insertion-to-abstraction mechanism transition with increasing energy.

As mentioned in Sec. III, the total DCS for $\mathrm{H}^{+}+\mathrm{H}_{2}$ $(v=0, j=0)$ at $E_{\text {coll }}=0.44 \mathrm{eV}$ presents nevertheless some departure from a complete symmetry with respect to the sideway direction. Figure 6 shows that there exists, in fact, some slight preference for the forward scattering direction. Interestingly, the EQM calculations we have carried out (not reported here) at the same collision energy on a different PES [the diatomics-in-molecule (DIM) PES previously used in Ref. 27] reveal a similar degree of asymmetry of the DCS, but with a preference, in this case, for the backward direction. It is also in the $\theta=180^{\circ}$ direction where the SQM approach gets the best agreement with the EQM result on the PES of Ref. 27. More dramatically, the pronounced peaks found for $j^{\prime}=0$ and $j^{\prime}=2$ in the forward and backward directions, respectively, of the $\mathrm{H}^{+}+\mathrm{H}_{2}(v=0, j=0) \rightarrow \mathrm{H}^{+}+\mathrm{H}_{2}$ $\left(v^{\prime}=0, j^{\prime}\right)$ reaction (see left top panels of Figure 7) are absent in the corresponding state-resolved DCSs on the DIM PES. In this sense, the above mentioned dependence on the collision energy found for the opacity functions obtained by means of the ACS approach supports the conclusion of extremely sensitive dynamical features on the collision energy and PES employed for the title exchange reaction. The comparison with experimental results ${ }^{40}$ would be probably a conclusive test for the present theoretical predictions and their possible dependence on the PES employed.

Comparison with the EQM results reported here reveals that the ACS approximation for the QWP calculation suggested in Ref. 30 and the SQM yield to a better description of the dynamics of the exchange $\mathrm{H}^{+}+\mathrm{H}_{2}(v=0, j=0)$ reaction as one might expect from conclusions of the previous study on the $\mathrm{H}^{+}+\mathrm{D}_{2}$ and $\mathrm{D}^{+}+\mathrm{H}_{2}$ processes. ${ }^{30}$ It would be of interest to test if a similar degree of agreement can be achieved for those two reactions. Work in this direction is now in progress.

The main limitation found in this work regarding the QCT approach is the underestimation of the reactivity at high values of the total angular momentum. However, in spite of this fact, the QCT method offers a quick alternative of calculating reaction observables, even for the title reaction, which in general are, at least, in qualitative agreement with more computationally demanding QM methods.

\section{ACKNOWLEDGMENTS}

Two of the authors (O.R. and T.G.L.) thank the Spanish Ministry of Education (MEC) for financial support through Grants Nos. FIS2004-02461 and CTQ2004-02415. One of the authors (T.G.L.) would like to acknowledge support from the program Ramón y Cajal of Spanish MEC and EU Grant No. MERG-CT-2004-513600. Two other authors (F.J.A. and L.B.) acknowledge financial support through MEC Grant No. CTQ2005-08493-C02-01 and Universidad ComplutenseComunidad de Madrid Grant No. 910729. Another author (N.B.) thanks a postdoctoral fellowship by Spanish MEC under the program "Estancias de jóvenes doctores y tecnólogos extranjeros en España." One of the authors (F.J.A.) thanks the financial support by the programme of "Sabáticos Complutenses" of the Universidad Complutense de Madrid. The EQM calculations were performed on a NEC-SX5 vector supercomputer, through a grant from the "Institut du Développement des Ressources en Informatique Scientifique" (IDRIS-CNRS) in Orsay (France).

${ }^{1}$ V. Kokoouline and C. H. Greene, Phys. Rev. Lett. 90, 133201 (2003).

${ }^{2}$ H. Kreckel, M. Motsch, J. Mikosch et al., Phys. Rev. Lett. 95, 263201 (2005).

${ }^{3}$ B. J. McCall, T. R. Geballe, K. H. Hinkle, and T. Oka, Science 279, 1910 (1998).

${ }^{4}$ B. J. McCall and T. Oka, J. Chem. Phys. 113, 3104 (2000).

${ }^{5}$ C. M. Lindsay and B. J. McCall, J. Mol. Spectrosc. 210, 60 (2001).

${ }^{6}$ A. Ichihara and K. Yokoyama, J. Chem. Phys. 103, 2109 (1995).

${ }^{7}$ A. Aguado, O. Roncero, C. Tablero, C. Sanz, and M. Paniagua, J. Chem. Phys. 112, 1240 (2000).

${ }^{8}$ C. Sanz, O. Roncero, C. Tablero, A. Aguado, and M. Paniagua, J. Chem. Phys. 114, 2182 (2001).

${ }^{9}$ O. Friedrich, A. Alijah, Z. R. Xu, and A. J. C. Varandas, Phys. Rev. Lett. 86, 1183 (2001).

${ }^{10}$ L. P. Viegas, M. Cernei, A. Alijah, and A. J. C. Varandas, J. Chem. Phys. 120, 253 (2004).

${ }^{11}$ P. Barragán, L. F. Errea, A. Macías, L. Méndez, I. Rabadán, A. Riera, J. M. Lucas, and A. Aguilar, J. Chem. Phys. 121, 11629 (2004).

${ }^{12}$ V. Kokoouline and F. Masnou-Seeuws, Phys. Rev. A 73, 012702 (2006).

${ }^{13}$ L. F. Errea, A. Macías, L. Méndez, I. Rabadán, and A. Riera, Phys. Rev. A 65, 010701 (2001)

${ }^{14}$ A. Ichihara, T. Shirai, and K. Yokoyama, J. Chem. Phys. 105, 1857 (1996).

${ }^{15}$ A. Ichihara, O. Iwamoto, and R. K. Janev, J. Phys. B 33, 4747 (2000).

${ }^{16}$ I. Last, M. Gilibert, and M. Baer, J. Chem. Phys. 107, 1451 (1997).

${ }^{17}$ V. G. Ushakov, K. Nobusada, and V. I. Osherov, Phys. Chem. Chem. Phys. 3, 63 (2001)

${ }^{18}$ T. Kusakabe, L. Pichl, R. J. Buenker, M. Kimura, and H. Tawara, Phys. Rev. A 70, 052710 (2004).

${ }^{19}$ H. Kamisaka, W. Bian, K. Nobusada, and H. Nakamura, J. Chem. Phys. 116, 654 (2002).

${ }^{20}$ D. Dai, C. C. Wang, S. A. Harich, H. Song, M. Hayes, R. T. Skodje, X. Wang, D. Gerlich, and X. Yang, Phys. Rev. Lett. 95, 013201 (2005). 
${ }^{21}$ H. Song, D. Dai, G. Wu, C. C. Wang, D. Gerlich, X. Yang, and R. T Skodje, J. Chem. Phys. 123, 074314 (2005).

${ }^{22}$ E. Wrede, L. Schnieder, K. Seekamp-Schnieder, B. Niederjohann, and K. H. Welge, Phys. Chem. Chem. Phys. 7, 1577 (2005).

${ }^{23}$ L. Schnieder, W. Meier, K. H. Welge, M. N. R. Ashfold, and C. M. Western, J. Chem. Phys. 92, 7027 (1990).

${ }^{24}$ X. Yang, Int. Rev. Phys. Chem. 24, 37 (2005).

${ }^{25}$ N. Markovic and G. D. Billing, Chem. Phys. 191, 247 (1995).

${ }^{26}$ M. Chajia and R. D. Levine, Phys. Chem. Chem. Phys. 1, 1205 (1999).

${ }^{27}$ T. Takayanagi, Y. Kurosaki, and A. Ichihara, J. Chem. Phys. 112, 2615 (2000).

${ }^{28}$ T.-S. Chu and K.-L. Han, J. Phys. Chem. A 109, 2050 (2005).

${ }^{29}$ R.-F. Lu, T.-S. Chu, and K.-L. Han, J. Phys. Chem. A 109, 6683 (2005).

${ }^{30}$ T. González-Lezana, A. Aguado, M. Paniagua, and O. Roncero, J. Chem. Phys. 123, 194309 (2005).

${ }^{31}$ E. J. Rackham, F. Huarte-Larrañaga, and D. D. Manolopoulos, Chem. Phys. Lett. 343, 356 (2001).

${ }^{32}$ E. J. Rackham, T. González-Lezana, and D. E. Manolopoulos, J. Chem. Phys. 119, 12895 (2003).

${ }^{33}$ R. T. Pack, J. Chem. Phys. 60, 633 (1974).

${ }^{34}$ P. McGuire and D. J. Kouri, J. Chem. Phys. 60, 2488 (1974).

${ }^{35}$ K. McLenithan and D. Secrest, J. Chem. Phys. 80, 2480 (1984).

${ }^{36}$ N. Markovic and G. D. Billing, Chem. Phys. Lett. 248, 420 (1996).

${ }^{37}$ N. Balucani, P. Casavecchia, L. Bañares, F. J. Aoiz, T. González-Lezana, P. Honvault, and J.-M. Launay, J. Phys. Chem. A 110, 817 (2006).

${ }^{38}$ N. Balucani, G. Capozza, E. Segoloni, A. Russo, R. Bobbenkamp, P. Casavecchia, T. González-Lezana, E. J. Rackham, L. Bañares, and F. J. Aoiz, J. Chem. Phys. 122, 234309 (2005).

${ }^{39}$ L. Bañares, F. J. Aoiz, T. González-Lezana, V. J. Herrero, and I. Tanarro, J. Chem. Phys. 123, 224301 (2005).

${ }^{40} \mathrm{X}$. Yang (private communication).

${ }^{41}$ P. Honvault and J.-M. Launay, in Theory of Chemical Reaction Dynamics, edited by G. Lendvay and A. Lagana (Kluwer Academic, Dordrecht 2004), p. 187

${ }^{42}$ P. Honvault and J. M. Launay, J. Chem. Phys. 111, 6665 (1999).

${ }^{43}$ P. Honvault and J. M. Launay, J. Chem. Phys. 114, 1057 (2001).

${ }^{44}$ B. Bussery-Honvault, P. Honvault, and J.-M. Launay, J. Chem. Phys. 115, 10701 (2001).

${ }^{45}$ P. Soldàn, M. T. Cvitas, J. M. Hutson, P. Honvault, and J.-M. Launay,
Phys. Rev. Lett. 89, 153201 (2002).

${ }^{46}$ G. Quéméner, P. Honvault, and J.-M. Launay, Eur. Phys. J. D 30, 201 (2004).

${ }^{47}$ M. T. Cvitas, P. Soldàn, J. M. Hutson, P. Honvault, and J.-M. Launay, Phys. Rev. Lett. 94, 033201 (2005).

${ }^{48}$ D. E. Manolopoulos, J. Chem. Phys. 85, 6425 (1986).

${ }^{49}$ B. R. Johnson, J. Comput. Phys. 13, 445 (1973).

${ }^{50}$ R. N. Zare, Angular Momentum, (Wiley, New York, 1988).

${ }^{51}$ Y. Huang, D. J. Kouri, and D. K. Hoffman, J. Chem. Phys. 101, 10493 (1994).

${ }^{52}$ V. A. Mandelshtam and H. S. Taylor, J. Chem. Phys. 103, 2903 (1995).

${ }^{53}$ Y. Huang, S. S. Iyengar, D. J. Kouri, and D. K. Hoffman, J. Chem. Phys. 105, 927 (1996).

${ }^{54}$ G.-J. Kroes and D. Neuhauser, J. Chem. Phys. 105, 8690 (1996).

${ }^{55}$ R. Chen and H. Guo, J. Chem. Phys. 105, 3569 (1996).

${ }^{56}$ S. K. Gray and G. G. Balint-Kurti, J. Chem. Phys. 108, 950 (1998).

${ }^{57}$ F. J. Aoiz, L. Bañares, and V. J. Herrero, J. Chem. Soc., Faraday Trans. 94, 2483 (1998).

${ }^{58}$ L. Bañares, F. J. Aoiz, P. Honvault, B. Bussery-Honvault, and J.-M. Launay, J. Chem. Phys. 118, 565 (2003).

${ }^{59}$ L. Bañares, F. J. Aoiz, P. Honvault, and J.-M. Launay, J. Phys. Chem. A 108, 1616 (2004)

${ }^{60}$ L. Bonnet and J.-C. Rayez, Chem. Phys. Lett. 277, 183 (1997).

${ }^{61}$ W. H. Miller, J. Chem. Phys. 52, 543 (1970).

${ }^{62}$ R. A. White and J. C. Light, J. Chem. Phys. 55, 379 (1971).

${ }^{63}$ P. Larrégaray, L. Bonnet, and J.-C. Rayez, J. Phys. Chem. A 110, 1552 (2006).

${ }^{64}$ W. B. Miller, S. A. Safron, and D. R. Herschbach, Discuss. Faraday Soc. 44, 108 (1967).

${ }^{65}$ D. O. Ham and J. L. Kinsey, J. Chem. Phys. 53, 285 (1970).

${ }^{66}$ I. G. Csizmadia, J. C. Polanyi, A. C. Roach, and W. H. Wong, Can. J. Chem. 47, 4097 (1969).

${ }^{67}$ J. R. Krenos, R. K. Preston, R. Wolfgang, and J. C. Tully, J. Chem. Phys. 60, 1634 (1973).

${ }^{68}$ D. Gerlich, U. Nowotny, Ch. Schlier, and E. Teloy, Chem. Phys. 47, 245 (1980).

${ }^{69}$ J. Krenos and R. Wolfgang, J. Chem. Phys. 52, 5961 (1970).

${ }^{70}$ R. K. Preston and J. C. Tully, J. Chem. Phys. 54, 4297 (1971). 